\title{
Mathematical Model
}

National Cancer Institute

\section{Source}

National Cancer Institute. Mathematical Model. NCI Thesaurus. Code C16870.

Theoretical representations that simulate the behavior or activity of systems, processes,

or phenomena. 